\title{
Progresso da ferrugem tropical do milho (Zea mays L.), sob diferentes tratamentos fungicidas*
}

\author{
Frank Magno da Costa $^{1,2}$, Modesto Barreto ${ }^{1}$, Érika Sayuri Maneti Koshikumo ${ }^{1,2}$, Fernandes Antonio de Almeida ${ }^{1,3}$
}

\begin{abstract}
${ }^{1}$ Departamento de Fitossanidade, Faculdade de Ciências Agrárias e Veterinárias/UNESP, CEP 14884 - 900, Jaboticabal,SP, Brasil; ${ }^{2}$ Bolsista de Mestrado CNPq; ${ }^{3}$ Aluno de Doutorado.

*Parte da dissertação de mestrado do primeiro autor.

Autor para correspondência: Frank Magno da Costa. fmcosta1@yahoo.com.br

Data de chegada: 27/02/2007. Aceito para publicação em: 21/01/2008
\end{abstract}

\section{RESUMO}

Costa, F. M.; Barreto, M.; Koshikumo, E.S.M.; Almeida. F.A. Progresso da ferrugem tropical do milho (Zea mays L), sob diferentes tratamentos fungicidas. Summa Phytopathologica, v.34, n.3, p.248-252, 2008

A ferrugem tropical, cujo agente causal é o fungo Physopella zeae (Mains) Cummins \& Ramachar vem se destacando por sua agressividade à cultura do milho. Objetivou-se, com este trabalho, ajustar modelos matemáticos às curvas de progresso desta doença sob a ação de misturas de triazóis e estrobilurinas sobre o fungo na cultura do milho. $O$ experimento foi conduzido em área experimental do Departamento de Fitossanidade da UNESP, Campus de Jaboticabal, no período de janeiro a maio de 2006, no delineamento de blocos casualizados completos com 6 tratamentos e 4 repetições. Os tratamentos utilizados foram (mL de p.c./ha): 1 - testemunha; 2 - epoxiconazole + pyraclostrobin, 500; 3 - epoxiconazole + pyraclostrobin, 750; 4 azoxystrobin + cyproconazole, 300 + óleo mineral parafínico $0,50 \%$, estes aplicados às plantas no estádio V8; 5 - azoxystrobin + cyproconazole, 300 + óleo mineral parafínico 0,50\%, este aplicado às plantas no estádio V8 e repetido quando esta encontrava-se no início do pendoamento e 6- azoxystrobin + cyproconazole, $450+$ óleo mineral parafínico $0,50 \%$, aplicado às plantas no estádio V8. Foram realizadas sete avaliações da severidade da doença com intervalos semanais, traçando-se a partir dos dados obtidos nestas avaliações as curvas de progresso da doença analisadas por meio do ajuste a três modelos matemáticos (monomolecular, logístico e de Gompertz). Calculou-se ainda a Área Abaixo das Curvas de Progresso da Doença (AACPD), resultante de cada tratamento. Procedeu-se à análise de variância dos dados e as médias foram comparadas pelo teste Tukey ao nível de $5 \%$ de probabilidade. Verificou-se que não houve diferença significativa entre os efeitos dos tratamentos 4,5 e 6 e esses proporcionaram menor AACPD e ainda que esses apresentaram eficiência de controle de $92 \%, 97 \%$ e $98 \%$, respectivamente. Pelos resultados obtidos, pode-se concluir que o modelo monomolecular foi o que melhor se ajustou aos dados. Não foi observado diferença significativa entre os efeitos dos tratamentos, no que se refere a produtividade.

Palavras-chave adicionais: Controle químico, Physopella zeae, AACPD.

\section{ABSTRACT}

Costa, F. M.; Barreto, M.; Koshikumo, E.S.M.; Almeida. F.A. Progress of the tropical rust of corn Zea mays L., under different fungicide treatments. Summa Phytopathologica, v.34, n.3, p.248-252, 2008

Among the three rusts diseases in the of corn crop, the tropical rust caused by Physopella zeae (Mains) Cummins \& Ramachar is highlighting by its aggressiveness. The objective of this study was to evaluate the fungicides mixtures action azoxystrobin + cyproconazole and epoxiconazole + pyraclosytrobin in the control of the tropical rust of corn in the culture of the crop. The experiment was carried out in the experimental area of the Department of crop protection of UNESP, Jaboticabal, São Paulo State, Brazil, during in the period of January to May of 2006. Were evaluated in the field plots were arranged in the complete randomized blocks, with 6 treatments and 4 replications. The used treatments were (mL of c.p./ha): 1-control; 2-epoxiconazole + pyraclostrobin 500,; 3-epoxiconazole + pyraclostrobin 750,; 4-azoxystrobin + cyproconazole $300+$ mineral oil $0,50 \%, ; 5$ - azoxystrobin + cyproconazole $300+$ mineral oil $0,50 \%$ and 6 azoxystrobin + cyproconazole $450+$ mineral oil 0,50\%. All the treatments fungicide spray were applied to maize at the stage V8, and the treatment 5 was replicated in the beginning of the tasseling. It was realized seven evaluations of the disease severity were performed every seven days. The curves of progress of the disease were analyzed by using three transformations models (monomolecular, logistico and of Gompertz). The AUDPC (Area Under the Disease Progress Curve) was calculated from which the analysis of variance was performed and the averages were compared by the Tukey test at the level of $5 \%$ of probability. The analysis no found difference significant statistics among the treatments 6 , 5 and 4, which these showed smaller AUDPC. We concluded that the monomolecular model it was best model adjusted to the data.

Additional keywords: Chemical control, Physopella zeae, AUDPC. 
A expansão das áreas cultivadas com a cultura do milho no Brasil, assim como a extensão das épocas de plantio e ainda a utilização de cultivares precoces com maior potencial de produção, algumas vezes mais suscetíveis às doenças, têm contribuído para o aumento na ocorrência de doenças foliares nessa cultura. No Brasil, os agentes etiológicos de doenças em milho causavam danos a esta cultura de maneira esporádica até o final da década de 80. A partir do início da década de 90, algumas doenças têm causado sensível redução na qualidade e na produtividade do milho, devido ao aumento da freqüência e severidade com que vêm ocorrendo $(5,12)$.

Dentre as doenças que acometem esta cultura, as ferrugens merecem destaque, pois estão classificadas entre as principais, haja vista, terem sido observadas na maioria das regiões produtoras, causando limitações a algumas destas, tais como danos diretos a planta, por redução da área fotossintetizadora, que pode acarretar redução na produção e/ou produtividade (13).

Em relação às três ferrugens que ocorrem no milho, a ferrugem tropical ou ferrugem branca, cujo agente causal é o fungo Physopella zeae (Mains) Cummins \& Ramachar, vem se destacando por sua maior adaptação a diferentes ambientes e pela disponibilidade de cultivares comerciais resistentes e ainda por sua agressividade. A primeira constatação dessa doença na América do sul data de 1944. No Brasil, os primeiros relatos de ocorrência da ferrugem tropical datam de 1976, e estes foram realizados no Estado do Espírito Santo. Desde então essa doença tem aumentado de forma epidêmica, ocorrendo em praticamente todas as regiões produtoras, principalmente nas regiões Centro-Oeste e Sudeste $(7,13)$.

Os sintomas desta doença apresentam-se como uredias de coloração creme a amarelada, ocorrendo principalmente em grupos pequenos, paralelamente às nervuras medindo cerca de $0,3-1,0 \mathrm{~mm}$ de comprimento. Estas aparecem em ambas as superfícies foliares e são cobertas pela epiderme, com exceção de um poro pequeno ou fenda no centro da lesão. Essas pústulas podem desenvolver-se e abranger o tecido foliar ao redor delas e sofrer uma alteração na coloração, tornandose amarelada a castanha e mudam para púrpura escuro, com uma área de $0,6 \mathrm{~cm}$ de diâmetro. Teliósporos enegrecidos são geralmente formados nestas manchas em grupos espalhados ao redor da urédia $(11,14)$. De acordo com Melching (9), Silva \& Mentem (16) o início da infecção ocorre em cerca de 7 a 9 dias após a inoculação à temperatura de $23-30{ }^{\circ} \mathrm{C}$, e em torno 10 dias após a inoculação à temperatura de 17-19 ${ }^{\circ} \mathrm{C}$. Esta doença tem recebido mais atenção devido ao fato de o fungo $P$. zeae apresentar maior adaptação em diferentes ambientes, possivelmente por ser menos exigente em umidade e temperatura quando comparado com os agentes causais da ferrugem polissora (Puccinia polysora) e ferrugem comum (Puccinia sorghi).

O conhecimento da epidemiologia de uma dada doença serve como base para que se apliquem os princípios de controle, haja vista, esses atuarem diretamente no ciclo das relações patógeno-hospedeiro, impedindo ou retardando o desenvolvimento dos eventos deste ciclo.

Visando avaliar os danos causados pelas ferrugens tropical e polissora Pinho et al. (12) observaram que a ferrugem tropical apresentou os maiores índices de severidade quando comparados com aqueles apresentados pela ferrugem polissora no município de Santa Helena de Goiás, muito embora os maiores níveis de danos tenham sido observados quando se fez a correlação parcial entre a AACPD e a produtividade de grãos considerando o efeito de Puccinia polysora e desconsiderando o efeito de $P$. zea. Estes autores, na época de avaliação de 80 dias após a semeadura, observaram uma redução em média de mais de 2,0 t/ha por acréscimo de uma unidade na nota de severidade, ou seja, $10 \%$ de área foliar afetada pela ferrugem tropical, evidenciando também, a necessidade de se recomendar, para essas regiões, somente cultivares com níveis aceitáveis de resistência.

O controle mais eficaz dessa doença é feito através do uso de resistência genética, muito embora sejam poucos os relatos de cultivares comercias com bons níveis de resistência à ferrugem tropical, sendo, algumas vezes, necessário entrar com medidas alternativas de controle, como por exemplo, o controle químico (6).

O acompanhamento da severidade da doença pode e deve ser inserido no estabelecimento de medidas de manejo integrado da ferrugem tropical na cultura do milho, pois auxilia a prever níveis futuros da doença em estudo, e indicar quando pulverizar com fungicidas, a dose mais eficiente e a alternância entre fungicidas, etc.

Considerando-se o baixo nível de resistência dos cultivares comerciais de milho à ferrugem tropical, objetivou-se, com este trabalho, acompanhar o progresso da doença ao longo do desenvolvimento da cultura sob a ação de misturas de triazóis e estrobilurinas no controle da doença.

\section{MATERIAL E MÉTODOS}

O experimento foi conduzido em área do Departamento de Fitossanidade da Faculdade de Ciências Agrárias e Veterinárias da Universidade Estadual Paulista, Campus de Jaboticabal, no período de janeiro a maio de 2006.

O início do ensaio se deu em 09 de Janeiro de 2006, quando realizouse a semeadura direta do híbrido 2B710, utilizando seis sementes por metro de sulco a uma profundidade de aproximadamente $5 \mathrm{~cm}$; o espaçamento entre linhas de cultivo foi de $90 \mathrm{~cm}$.

O delineamento experimental utilizado foi o de blocos casualizados completos, com seis tratamentos e quatro repetições. As parcelas foram constituídas de dez linhas de onze metros lineares cada, totalizando $100 \mathrm{~m}^{2}$ nas quais as duas linhas centrais foram consideradas como área útil do experimento.

Os tratamentos fungicidas utilizados encontram-se expressos na Tabela 1, todos foram aplicados no estádio V8, sendo que o Tratamento 5 foi repetido no início do pendoamento. Para a aplicação dos tratamentos utilizou-se um pulverizador costal pressurizado com $\mathrm{CO} 2$ e volume de calda de $200 \mathrm{~L} / \mathrm{ha}$.

A primeira aplicação foi realizada no dia 06/03/2006, quando a planta encontrava-se aos 56 dias após a semeadura (DAS). Os dados meteorológicos registrados para este dia foram: temperatura média $25,8^{\circ} \mathrm{C}$ e umidade relativa média do ar $79 \%$ (8). Na segunda aplicação apenas o tratamento 5 foi empregado, visando determinar se a repetição deste implicaria em maior eficiência de controle e maior produtividade. Este se deu 15 dias após a primeira aplicação, quando as plantas atingiam o início do pendoamento aos 71 (DAS), ou seja, no dia 21/03/ 2006 , em que a temperatura média foi de $25,9^{\circ} \mathrm{C}$ e a umidade relativa média do ar foi de $84 \%$ (8).

Para as avaliações, foram marcadas dez plantas ao acaso por parcela, cinco plantas em cada linha da área útil das parcelas do experimento, com a finalidade de acompanhar o progresso da doença sempre nas mesmas plantas. Um total de 7 avaliações foram realizadas no ensaio, com intervalos semanais entre elas. A severidade da ferrugem tropical foi avaliada em 10 folhas individualmente por planta. A enumeração das folhas para as avaliações se deu desconsiderando as duas primeiras folhas baixeiras, ou seja, as folhas mais velhas. A partir destas, a folha seguinte foi considerada como sendo a primeira, para fins de avaliação dos sintomas e acompanhamento do progresso da doença durante o desenvolvimento da planta. As demais folhas foram enumeradas em ordem crescente até a décima folha. A escala de notas utilizada para 
Tabela 1. Tratamentos fungicidas utilizados, ingrediente ativo, produto comercial, dose (mL de P.C./ha), estádio da planta de milho e época de aplicação. Jaboticabal-SP, 2006

\begin{tabular}{|c|c|c|c|c|}
\hline Tratamentos & Produtocomercial & $\operatorname{Dose}(m L$ de P.C./ha) & Estádio daplanta & Época de aplicação \\
\hline Testemunha & - & - & - & - \\
\hline epoxiconazole+pyraclostrobin & Ópera & 500 & V8 & $06 / 03 / 2006$ \\
\hline epoxiconazole+pyraclostrobin & Ópera & 750 & V8 & $06 / 03 / 2006$ \\
\hline azosxystrobin+cyproconazole +óleo mineral parafínico & Priori Xtra +Nimbus & $300+0,5 \%$ & V8 & $06 / 03 / 2006$ \\
\hline azosxystrobin cyproconazole +óleo mineral parafínico & Priori Xtra + Nimbus & $300+0,5 \%$ & V8 /I.P.* & $06 / 03 / 2006+21 / 03 / 2006$ \\
\hline azosxystrobin+cyproconazole +óleo mineral parafínico & Priori Xtra + Nimbus & $450+0,5 \%$ & V8 & $06 / 03 / 2006$ \\
\hline
\end{tabular}

*início do pendoamento

avaliar a severidade foi adaptada de Azevedo (2) variando de 0 a 6 , correspondendo à nota 0 - $0 \%$ ou ausência de sintomas; 1 - 0,1 a $3 \%$; $2-3,1$ a $6 \% ; 3-6,1$ a $12 \% ; 4-12,1$ a $25 \% ; 5-25,1$ a $50 \%$ e 6 - mais de $50 \%$ da área foliar lesionada. O início das avaliações se deu três dias após a primeira aplicação dos tratamentos, ou seja, no dia 09/03/ 2006, quando a planta estava com 59 DAS, sendo este dia considerado como o tempo zero de avaliação e continuou a cada sete dias até se completar a sétima avaliação no dia 20/04/2006, com a planta aos 101 DAS. Ao surgirem os primeiros sintomas, folhas de plantas não marcadas foram colhidas e conduzidas ao Laboratório de Fitopatologia da UNESP, Campus de Jaboticabal, a fim de se realizar a diagnose através da observação de estruturas do patógeno com o auxílio de microscópio estereoscópico e de microscópio óptico.

No dia 27/05/2006, foi feita a colheita das espigas das plantas marcadas. Inicialmente foram debulhadas manualmente para pesagem, obtendo-se o peso total $(\mathrm{kg})$ de cada amostra e o peso $(\mathrm{g})$ de 100 sementes por amostra.

Para uma melhor representação e compreensão da epidemia no patossistema em estudo, ajustaram-se os modelos para as curvas de progresso da ferrugem tropical, através do programa Microsoft Excel $2003^{\circledR}$, por meio da plotagem dos valores de severidade (variável dependente) obtidos nesta pesquisa em função do tempo (variável independente). Os modelos matemáticos mais utilizados em epidemiologia foram ajustados aos dados: o modelo monomolecular

Tabela 2. Valores dos coeficientes de determinação $\left(\mathrm{R}^{2}\right)$, obtidos pela regressão linear entre os valores previstos e observados para cada transformação e folha e tratamentos usados na escolha do modelo que melhor se ajustou aos dados. Jaboticabal-SP, 2006.

\begin{tabular}{|c|c|c|c|c|c|c|c|c|}
\hline Tratamentos" & Modelo & Folha 1 & Folha 2 & Folha 3 & Folha 4 & Folha 5 & Folha 6 & Médias \\
\hline \multirow{3}{*}{1} & Monomolecular & $0,048^{* * *}$ & 0,541 & 0,890 & 0,892 & 0,946 & 0,938 & 0,709 \\
\hline & Logístico & 0,004 & 0,143 & 0,465 & 0,452 & 0,527 & 0,496 & 0,348 \\
\hline & Gompertz & 0,001 & 0,251 & 0,610 & 0,607 & 0,704 & 0,687 & 0,477 \\
\hline \multirow{3}{*}{2} & Monomolecular & 0,774 & 0,958 & 0,940 & 0,965 & 0,941 & 0,915 & 0,915 \\
\hline & Logístico & 0,414 & 0,597 & 0,542 & 0,797 & 0,654 & 0,670 & 0,612 \\
\hline & Gompertz & 0,536 & 0,732 & 0,686 & 0,888 & 0,777 & 0,779 & 0,733 \\
\hline \multirow{3}{*}{3} & Monomolecular & 0,742 & 0,881 & 0,955 & 0,959 & 0,937 & 0,957 & 0,905 \\
\hline & Logístico & 0,352 & 0,456 & 0,567 & 0,564 & 0,520 & 0,638 & 0,516 \\
\hline & Gompertz & 0,472 & 0,594 & 0,719 & 0,710 & 0,682 & 0,786 & 0,661 \\
\hline \multirow{3}{*}{4} & Monomolecular & 0,850 & 0,921 & 0,923 & 0,899 & 0,375 & 0,903 & 0,812 \\
\hline & Logístico & 0,612 & 0,696 & 0,607 & 0,801 & 0,550 & 0,826 & 0,682 \\
\hline & Gompertz & 0,699 & 0,762 & 0,696 & 0,847 & 0,543 & 0,868 & 0,736 \\
\hline \multirow{3}{*}{5} & Monomolecular & 0,373 & 0,720 & 0,945 & 0,796 & 0,750 & 0,750 & 0,722 \\
\hline & Logístico & 0,135 & 0,443 & 0,715 & 0,539 & 0,532 & 0,744 & 0,518 \\
\hline & Gompertz & 0,181 & 0,502 & 0,789 & 0,589 & 0,579 & 0,757 & 0,566 \\
\hline \multirow{3}{*}{6} & Monomolecular & 0,192 & 0,905 & 0,375 & 0,750 & 0,750 & 0,750 & 0,620 \\
\hline & Logístico & 0,047 & 0,618 & 0,279 & 0,601 & 0,601 & 0,744 & 0,482 \\
\hline & Gompertz & 0,074 & 0,703 & 0,295 & 0,630 & 0,630 & 0,757 & 0,515 \\
\hline \multirow{3}{*}{ Maior $\mathrm{R}^{2}$} & Monomolecular & 0,850 & 0,958 & 0,955 & 0,965 & 0,946 & 0,957 & 0,915 \\
\hline & Logístico & 0,612 & 0,696 & 0,715 & 0,801 & 0,654 & 0,826 & 0,682 \\
\hline & Gompertz & 0,699 & 0,762 & 0,789 & 0,888 & 0,777 & 0,868 & 0,736 \\
\hline
\end{tabular}

*Tratamento 1- Testemunha; Tratamento 2 - epoxiconazole + pyraclostrobin 500; Tratamento 3 - epoxiconazole + pyraclostrobin 750 ; Tratamento 4 - azoxystrobin + cyproconazole 300 + óleo mineral parafínico 0,50\% V8; Tratamento 5 - azoxystrobin + cyproconazole 300 + óleo mineral parafínico $0,50 \%$ V8 / IP; Tratamento 6 - azoxystrobin + cyproconazole $450+$ óleo mineral parafínico $0,50 \%$ V8.

** coeficiente de determinação $\left(\mathrm{R}^{2}\right)$ 


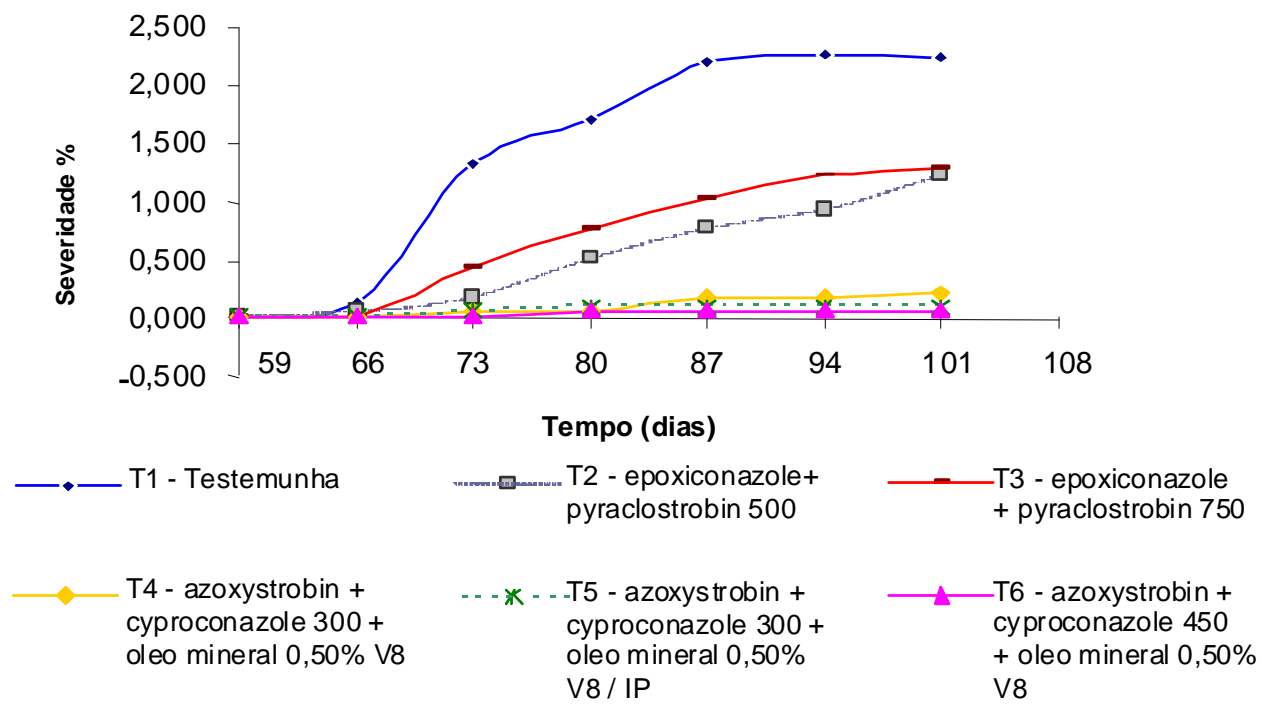

Figura 1. Curvas de progresso da ferrugem tropical do milho expresso em porcentagem de área foliar lesionada em função do tempo. Jaboticabal-SP, 2006.

$Y=b_{I} *\left(1-b_{I}{ }^{*} \exp (-r t)\right)$, o modelo logístico $Y=b_{I} /\left(1+b_{2}{ }^{*} \exp (-r t)\right)$ e o modelo de Gompertz $Y=b_{1}{ }^{*} \exp \left(-b_{2} \exp (-r t)\right)$, em que $Y$ é a severidade da doença, $b_{1}$ representa a assíntota máxima estimada pelo modelo, $b_{2}$ é a constante de integração igual a $1-y_{0}\left(y_{0}=\right.$ inóculo inicial $), r$ representa a taxa de progresso da doença e $t$ o tempo.

Os valores de severidade da doença obtidos nas diferentes parcelas em cada tratamento, e transformados em proporção de doença foram plotados versus o tempo e expressos em curva de progresso da doença, a fim de representar a epidemia. Com os dados de severidade em cada avaliação calculou-se, para cada parcela, os valores da área abaixo da curva de progresso da doença (AACPD) através do programa AVACPD versão1.1, fevereiro de 1992, desenvolvido por Torres \& Ventura (17), através da equação citada por Shaner \& Finney (15).

$$
\begin{aligned}
& A A C P D=\sum_{i=1}^{n}\left[\left(Y_{i+1}+Y_{i}\right) / 2\right] *\left[\left(T_{i+1}-T_{i}\right)\right] \\
& \text { Em que: } \\
& n \text {-é o número de observações. } \\
& Y_{i} \text { - é a severidade da doença na "i"-ésima observação; } \\
& T_{\mathrm{i}} \text { - é o tempo em dias na "i"-ésima observação; }
\end{aligned}
$$

Para a escolha do modelo que melhor se ajustou aos dados, levouse em consideração o maior valor do coeficiente de determinação $\left(R^{2}\right)$, obtido da regressão linear entre os valores previstos (variável dependente) e observados (variável independente). Os dados obtidos da AACPD foram transformados em $\sqrt{x+0,5}$ para atenderem aos pressupostos básicos da análise de variância, e os mesmos foram submetidos ao teste $\mathrm{F}$ na análise de variância e as médias comparadas pelo teste Tukey ao nível de 5\% de significância.

\section{RESULTADOS E DISCUSSÃO}

O grau de ajuste de cada modelo foi avaliado em função dos valores do coeficiente de determinação $\left(\mathrm{R}^{2}\right)$, e estão apresentados na Tabela 2 .

Observa-se na Tabela 2 que os coeficientes de determinação foram obtidos apenas para as folhas de 1 a 6 , pois nas folhas de 7 a 10, não se observou a presença de pústulas da ferrugem tropical. Com base nestes dados observa-se que a transformação monomolecular foi a que melhor se ajustou aos dados experimentais de progresso da doença em estudo, pois proporcionou um maior $\left(\mathrm{R}^{2}\right)$ em $91,66 \%$ dos casos, em relação aos modelos matemáticos de Gompertz e o logístico, que por sua vez proporcionaram maior $\left(\mathrm{R}^{2}\right)$ em apenas $5,55 \%$ e $2,78 \%$ dos casos, respectivamente. Isto pode ter ocorrido provavelmente as condições climáticas registradas para a época em que foi conduzido o experimento ter sido atípica, o que implicou em baixo inóculo inicial, que pode ter interferido no desenvolvimento normal da doença e ainda ter sido o modelo monomolecular o que melhor se ajustou aos dados. Com isto foi possível observar também que a velocidade de aumento da ferrugem tropical foi proporcional à quantidade de inóculo previamente existente (inóculo inicial) e à taxa de infecção. Estas observações estão de acordo com Bergamim Filho (3). Este modelo tem sido recomendado para o ajuste de dados de doenças com período de incubação variável ( 3 e 4) como é o caso da ferrugem tropical cujo período de incubação é mais dependente da temperatura (9). Observase ainda que para as seis folhas nas quais se obteve o coeficiente de determinação, neste ensaio, a folha 4 foi a que apresentou o maior $\mathrm{R}^{2}$.

As curvas de progresso da ferrugem tropical, para cada um dos seis tratamentos encontram-se representadas na Figura 1. Observa-se que o maior progresso da doença se deu a partir dos 66 DAS para todos os tratamentos, muito embora o máximo de severidade $\left(y_{\max }\right)$ tenha ocorrido na parcela referente ao tratamento Testemunha. Os tratamentos epoxiconazole + pyraclostrobin, 500 e epoxiconazole + pyraclostrobin, 750 também permitiram evidenciar o progresso da doença, apresentando severidade média de 0,504 e 0,664 \% da área foliar lesionada, respectivamente. O padrão assíntótico destas curvas é característico dos modelos monomolecular, logístico e de Gompertz, de acordo com Bergamin Filho (3). Para os Tratamentos 4 azoxystrobin + cyproconazole, $300+$ óleo mineral parafínico 0,$50 ; 5$ - azoxystrobin + cyproconazole, $300+$ óleo mineral parafínico 0,50 $\%$ e 6 - azoxystrobin + cyproconazole, 450 + óleo mineral parafínico $0,50 \%$, as severidades máximas $\left(y_{\max }\right)$ observadas foram: $(0,198 ; 0,085$ e $0,048 \%$ ), respectivamente, concordando com os dados obtidos por Pereira (10).

Os valores médios de severidade e AACPD, assim como o peso de grãos (kg/parcela) e a porcentagem de controle (eficiência dos efeitos dos tratamentos) são expressos na Tabela 3.

Pode-se observar que houve efeito significativo para os efeitos dos tratamentos sobre a doença. Os tratamentos fungicidas 4 azoxystrobin + cyproconazole, 300 + óleo mineral parafínico 0,50; 5 
Tabela 3. Severidade da ferrugem tropical, área abaixo da curva de progresso da doença (AACPD), produção de grãos e porcentagem de controle em relação à testemunha, Jaboticabal-SP, 2006.

\begin{tabular}{|c|c|c|c|c|}
\hline Tratamentos & $\begin{array}{l}\text { Severidade } \\
\text { média }\end{array}$ & AACPD & $\begin{array}{l}\text { Produção } \\
\text { de grãos } \\
\text { (kg/parcela) }\end{array}$ & $\begin{array}{l}\text { Eficiência de } \\
\text { controle } \\
\text { (ABOTT, 1925) }\end{array}$ \\
\hline Testemunha & 1,409 & $7,7610 \mathrm{a}^{1,2}$ & $1,675 \mathrm{a}^{1}$ & $0,00^{3}$ \\
\hline epoxiconazole + pyraclostrobin 750 & 0,664 & $5,2522 \mathrm{ab}$ & 1,785 a & 45,16 \\
\hline epoxiconazole + pyraclostrobin 500 & 0,504 & $4,4754 \quad b$ & $1,760 \mathrm{a}$ & 48,39 \\
\hline azoxystrobin + cyproconazole 300 + óleo mineral parafínico $0,50 \%$ V8 & 0,080 & 1,6800 & 1,865 a & 91,91 \\
\hline azoxystrobin + cyproconazole 300 + óleo mineral parafínico $0,50 \% \mathrm{~V} 8$ / IP & 0,048 & 1,2762 & 1,715 a & 96,77 \\
\hline 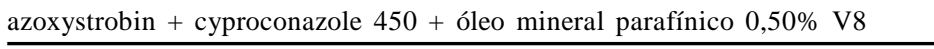 & 0,021 & 1,0408 & $1,755 \mathrm{a}$ & 98,37 \\
\hline Médias & 0,454 & -- & -- & -- \\
\hline $\mathrm{DMS}^{4}$ & - & 2,526 & 0,328 & - \\
\hline F Tratamentos & - & $24,17^{* *}$ & $0,82^{\mathrm{NS}}$ & - \\
\hline $\mathrm{CV}(\%)$ & -- & 30.67 & 8,11 & -- \\
\hline
\end{tabular}

${ }^{1}$ Médias seguidas de mesma letra não diferem entre si pelo teste Tukey a $1 \%$ de probabilidade; ${ }^{2}$ Dados transformados em $\sqrt{x+0,5} ;{ }^{3}$ Eficiência dos tratamentos em relação à testemunha mediante a utilização da fórmula de ABBOTT, (1925); ${ }^{4}$ Resumo da análise de variância; ** Significativo a $1 \%$; ${ }^{\text {NS }}$ Não significativo.

- azoxystrobin + cyproconazole, $300+$ óleo mineral parafínico 0,50 $\%$ e 6 - azoxystrobin + cyproconazole, 450 + óleo mineral parafínico $0,50 \%$ proporcionaram menores valores de $\mathrm{AACPD}$, não diferindo significativamente entre si. O maior valor de AACPD ocorreu nas parcelas testemunha e que não diferiu significativamente do obtido no tratamento 3 - epoxiconazole + pyraclostrobin, 750 e este do tratamento 2 - epoxiconazole + pyraclostrobin, 500. As maiores eficiências no controle da ferrugem tropical foram observadas nas parcelas referentes aos tratamentos que incluíram azoxystrobin + cyproconazole independente da época de aplicação. Para peso (kg/parcela) não houve diferença significativa entre os efeitos dos tratamentos fungicidas analisados. Depreende-se que a doença, nessas condições, época e local ocorreu com severidade abaixo do limiar de dano, pois a mesma não interferiu na produtividade da cultura.

Pelos resultados do presente trabalho conclui-se que na época e sob as condições em que foi realizado o experimento, devido a baixa severidade da doença observada, a mesma não interferiu na produtividade, fato este atribuído provavelmente, a quantidade de inoculo inicial do patógeno ter sido muito baixa.

\section{AGRADECIMENTOS}

Ao CNPq pela concessão da bolsa de mestrado.

\section{REFERÊNCIAS BIBLIOGRÁFICAS}

1. Abbott, W.S. A method of computing the effectiveness of an insecticide. Journal of Economic Entomology, College Park, v. 18, p. 265-267, 1925 .

2. Azevedo, L.A.S. Manual de quantificação de doenças de plantas. São Paulo, 1997. 114 p.

3. Bergamim Filho, A. Curvas de progresso da doença. In: Bergamim Filho, A.; Kimati, H.; Amorim, L. Manual de fitopatologia. São Paulo: Ceres, 1995. v. 1, cap. 30, p. 602-626.

4. Bergamim Filho, A.; Hau, B.; Amorim, L.; Laranjeira, F.F. Análise espacial de epidemias. Revisão anual de patologia de plantas. Passo Fundo: EMBRAPA, v. 10, p. 155-218, 2002.

5. Carvalho, R.V. Resistência do milho a Physopella Zeae (Mains Cummins \& Ramachar), agente causal da ferrugem tropical. 1995. 83f. Dissertação (Mestrado em Fitopatologia)-Escola Superior de Agricultura de Luiz de Queiroz, Uni- versidade de São Paulo, Piracicaba.

6. Dudienas C.; Sawazaki, E. Paterniani, M.E.A.G.; Galvão, J.C.C.; De Sordi, G.; Pereira, J. Comportamento de cultivares de milho, em condições de campo, quanto à resistência a Physopella zeae. Summa Phytopathologica, Jaboticabal, v.23, n.3/4, p. 259 262, 1997.

7. Figueiredo, M.B.; Hennen, J.F. Detection of telia of Physopella zeae in Piracicaba, state of São Paulo. Summa Phytopathologica, Jaguariúna, v.21, n.1, p.38-40, 1995.

8. INPE - Instituto Nacional de Pesquisas Espaciais. Disponível em: <http://tempo.cptec.inpe.br:9080/PCD>. Acesso em: 15 jan. 2007.

9. Melching, J.S. Corn rusts: types, races and destructive potencial. In: Annual corn and sorghum research conference, 30., 1975, Washington. Proceedings. Washington: American seed trade association 1975. p.90-115.

10. Pereira, O.A.P. Situação atual de doenças da cultura do milho no Brasil e estratégias de controle. In: Encontro sobre temas de genética e melhoramento, 1995, Piracicaba. Anais... Piracicaba: ESALQ, 1995. v.12. p.25-30.

11. Pereira, O.A.P. Doenças do milho. In: Kimati, H.; Amorin, L.; Bergamin Filho, A.; Camargo, L.E.A.; Rezende, J.A.M. Manual de fitopatologia. 3.ed. São Paulo: Ceres, 1997. v.2, p.538-555.

12. Pinho, R.G.V.; Ramalho, M.A.P.; Silva, H.P.; Resende, I.C.; Pozar, G. Danos causados pelas ferrugens polissora e tropical do milho. Fitopatologia Brasileira, Brasília, DF, v. 24, n.3, p. 400-409, 1999.

13. Pinho, R.G.V.; Ramalho, M.A.P.; Resende, I.C.; Pozar, G.; Olivatto, A.N.D. Controle genético da resistência do milho às ferrugens polissora e tropical. Fitopatologia Brasileira, Brasília, DF, v. 24, n.3, p. 394-399, 1999.

14. Reis, E.M.; Casa, R.T.; Bresolin, A.C.R. Manual de diagnose e controle de doenças do $\mathbf{m}$ ilho. 2.ed. Lages: Graphel, 2004. v. 2, $141 \mathrm{p}$.

15. Shaner, G.; Finney, R.E. The effect of nitrogen fertilization on the expression of slow - mildewing resistance in Knox wheat. Phytopathology, St. Paul, v.67, n.8, p.1051-1056, 1977.

16. Silva, H.P.; Mentem, J.O.M. Manejo integrado de doenças na cultura do milho. In: Fancelli, A.L.; Dourado-Neto, D. Tecnologia da produção de milho. Piracicaba: Publique, 1997. p. 4056.

17. Torres, J.C.; Ventura, J.A. AVACPD: um programa para calcular a área e o volume abaixo da curva de progresso da doença. Fitopatologia Brasileira, Brasília, DF, v.16, Suplemento, p.8, 1991. 